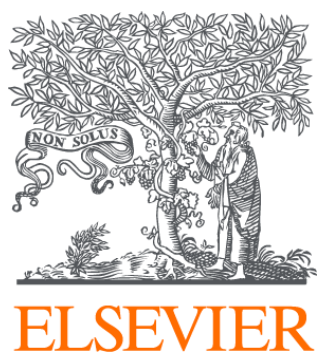

Since January 2020 Elsevier has created a COVID-19 resource centre with free information in English and Mandarin on the novel coronavirus COVID-

19. The COVID-19 resource centre is hosted on Elsevier Connect, the company's public news and information website.

Elsevier hereby grants permission to make all its COVID-19-related research that is available on the COVID-19 resource centre - including this research content - immediately available in PubMed Central and other publicly funded repositories, such as the WHO COVID database with rights for unrestricted research re-use and analyses in any form or by any means with acknowledgement of the original source. These permissions are granted for free by Elsevier for as long as the COVID-19 resource centre remains active. 


\title{
The powerful immune system against powerful COVID-19: A hypothesis
}

\author{
Farzad Taghizadeh-Hesary ${ }^{\mathrm{a}, *}$, Hassan Akbari ${ }^{\mathrm{a}, \mathrm{b}}$ \\ ${ }^{a}$ Shahid Beheshti University of Medical Sciences, Tehran, Iran \\ ${ }^{\mathrm{b}}$ Traditional Medicine School, Tehran University of Medical Sciences, Tehran, Iran
}

A R T I C L E I N F O

\section{Keywords:}

COVID-19

ATP

Immune dysfunction

\begin{abstract}
A B S T R A C T
On March 11, 2020, the World Health Organization declared the coronavirus outbreak a pandemic. Since December 2019, the world has experienced an outbreak of coronavirus disease 2019 (COVID-19). Epidemiology, risk factors, and clinical characteristics of patients with COVID-19 have been reported but the factors affecting the immune system against COVID-19 have not been well described. In this article, we provide a novel hypothesis to describe how an increase in cellular adenosine triphosphate (c-ATP) can potentially improve the efficiency of innate and adaptive immune systems to either prevent or fight off COVID-19.
\end{abstract}

\section{Background}

Today, the rapid outbreak of Corona Virus Disease 2019 (COVID-19 or SARS-CoV-2) is the leading health issue. There is a paucity of studies investigating the factors affecting immune response to COVID-19. In addition, there has been no detailed report for this immune response. Given the genomic similarity of 79\% with Severe Acute Respiratory Syndrome coronavirus (SARS-CoV), nearly the same reaction to the immune system is expected for COVID-19 [1]. In response to SARS-CoV, both innate and adaptive immune systems are involved. SARS-CoV applies several mechanisms to overcome the immune response. First, it inhibits the rapid expression of interferon type 1 (IFN-1) [2]. IFN-1 is known as the "initial alarm" upon encounter with the virus that modulates the immune cells to the so-called "antiviral state". Moreover, SARS-CoV interferes with IFN-1 signaling through inhibition of STAT-1 phosphorylation [3]. The third defensive mechanism of SARS-CoV is immune exhaustion through exaggerated and prolonged IFN-1 production by plasmacytoid dendritic cells (pDCs). This process leads to the influx of activated neutrophils and inflammatory monocytes/macrophages, that in turn, results in lung immunopathology (e.g. acute respiratory distress syndrome) [4]. Finally, the resulted so-called "cytokine storm" further weakens the immune system through IFN-1mediated $\mathrm{T}$ cell apoptosis [5]. In this article, we aim to provide a new hypothesis to describe how the repletion of cellular adenosine triphosphate (c-ATP) can promote immunity against COVID-19. Thereafter, we justify the current knowledge regarding the characteristics of COVID-19 infection by our hypothesis and give several approaches to improve the c-ATP.

\section{The hypothesis}

Considering the pivotal role of ATP in cellular function, c-ATP depletion can lead to cellular dysfunction [6]. Immune cells are not an exception. In this article, c-ATP is the index of cellular energy.

\section{Evaluation of the hypothesis}

Here, we show how c-ATP repletion can counteract with defensive mechanisms of COVID-19 and promote the immune system to the enhancement pathway.

\section{ATP facilitates IFN production}

COVID-19 interferes with a rapid rise in IFN-1. Therefore, it deactivates the so-called "initial alarm" of the innate immune system, by unknown mechanisms. This facilitates its replication. Zhang et al. have demonstrated that enhancement in the c-ATP can reverse this process. This occurs by the facilitation of IFN secretion through P38/JNK/ATF-2 signaling pathway [7]. Therefore, ATP-depleted cells are more susceptible to this effect of COVID-19.

\section{ATP facilitates IFN signaling}

Following IFN-1 secretion, fundamental changes occur in the immune cells that transform them into the so-called "antiviral state". One of the signaling pathways that take part in this process is the JAK/STAT pathway. JAKs are ATP-dependent enzymes that are bound to the cytoplasmic regions of cytokine receptors. Following attachment of IFN-1

\footnotetext{
* Corresponding author.

E-mail address: f_taghizadeh@sbmu.ac.ir (F. Taghizadeh-Hesary).
} 
to the cytokine receptor, JAK activates the STAT through trans-phosphorylation [8]. Obviously, c-ATP depletion interferes with this process and further impairs transformation to "antiviral state".

\section{ATP prevents the cytokine storm}

Following deactivation of "initial alarm", COVID-19 easily proliferates in-situ. Among the passive host-cells, there are exceptions that can react to the COVID-19, the pDCs. They detect the virus by toll-like receptor 7 (TLR-7). Upon attachment to viral nucleic acids, TLR7 induces profound IFN-1 expression. This response recruits other immune cells and causes massive local inflammation [9]. At first glance, this robust immune response is beneficial for the elimination of COVID-19. However, two factors prevent it. First, impairment of IFN-1 signaling results in impairment of immune cell transformation to the "antiviral state". Therefore, they are not so effective in eliminating existing viruses [8]. Second, persistence profound inflammatory responses may lead to immune exhaustion [4]. The depletion of c-ATP can potentially enhance these detrimental processes in the following ways. In 2016, Rebbapragada et al. demonstrated the effect of ATP in the function of TLR7 by controlling the endo-lysosomal PH. They showed that ATPdepletion can increase the endo-lysosomal $\mathrm{PH}$ and improve the efficacy of TLR7. Therefore, ATP-depletion can potentially enhance profound IFN-1 secretion in this phase. Secondly, ATP-depletion can potentially prone the recruited immune cells to earlier exhaustion against COVID19. Therefore, one may conclude that ATP-repletion can prevent the socalled "cytokine storm" and improve the cellular energy to better counteract with COVID-19.

\section{ATP prevents T-cell apoptosis}

Channappanavar et al. demonstrated that COVID-19 can promote Tcells to IFN-induced apoptosis, resulting in reduced numbers of virusspecific CD8 and CD4 T-cells [5]. From the perspective of cellular energy, this process potentially occurs through IFN-mediated T-cell activation that results in c-ATP depletion. In line with this hypothesis, Perl et al. have shown that following IFN- $\gamma$ stimulation, mitochondrial hyperpolarization and ATP depletion occurs in T-cells that results in apoptosis [10]. Therefore, ATP-repletion can potentially prevent T-cell apoptosis following "cytokine storm".

\section{Empirical data}

In the following section, we use our hypothesis to demonstrate why specific groups of people are more susceptible to be infected with COVID-19 and why they have a worse prognosis.

\section{Elderly population}

The case-fatality rate of COVID-19 is the highest (14.8\%) in elderlypopulation. In contrast, children have the lowest risk for both infection and mortality rates [11]. This difference can be demonstrated from the cellular energy aspect. Aging may potentially attenuate the respiratory capacity of mitochondria. This condition may be either due to impairment of peroxisome proliferator-activated receptor-gamma coactivator$1 \alpha$ (PGC- $1 \alpha$ ) or age-related accumulation of mitochondrial DNA mutations [12]. Moreover, aging can wane the ability of immune cells to secrete IFN following viral infection [13]. As noted earlier, this may be due to ATP-depletion. Therefore, one can conclude that a gradual decline in prognosis with age may rely on a gradual decrease in c-ATP.

\section{Tobacco smokers}

The risk of long-lasting and serious COVID-19 infection is more among tobacco smokers. Apart from a direct effect on lung parenchyma and a decrease in pulmonary capacity, tobacco smoke can potentially induce immune dysfunction through a decrease in the ATP content of immune cells. This can be due to nicotine-induced mitochondrial dysfunction [14]. The resultant ATP-depletion increases the risk of immune dysregulation by COVID-19 (refer to the aforementioned defensive mechanisms of COVID-19).

Male gender

While men and women have the same susceptibility to COVID-19, men are more prone to higher morbidity and mortality independent of age [15]. This difference can be justified by the cell energy hypothesis. Estrogens (as the main sex steroid of females) are potent stabilizers of ATP production during oxidative stress (e.g. during COVID-19-induced inflammation) [16]. Therefore, it seems that women are more capable to maintain the c-ATP of their immune cells during the immune response to COVID-19. With this notion in mind, men are more susceptible to immune dysregulation following COVID-19 infection.

\section{Serious chronic medical conditions}

Recent reports have highlighted some chronic illnesses that increase the mortality of COVID-19. They include underlying conditions such as hypertension, diabetes, coronary heart disease, chronic obstructive lung disease, cancer, and chronic kidney disease [17]. Apart from a decline in cardiovascular reserve, the effect of these chronic conditions on the prognosis of COVID-19 can be justified by our hypothesis. Human cells need nutrients (including glucose, free fatty acids, essential amino acids, and $\mathrm{O}_{2}$ ) to maintain their c-ATP level. The aforementioned illnesses impede the regular distribution of the nutrients secondary to compromising the function and structure of small and large vessels. Therefore, the human cells (including in-situ immune cells) confront ATP-depletion and results in further immune dysregulation (as mentioned above).

\section{Approaches to improvement in c-ATP}

In light of these considerations, the c-ATP level can potentially be considered as a crucial component in the infectivity and prognosis of COVID-19. With enhancing the c-ATP, improvement in both innate and adaptive immune systems is expected. Moreover, an increase in c-ATP can potentially have either preventive or therapeutic effects. The preventive effect through activation of initial IFN-1 secretion and signaling, as "initial alarm" of the innate immune system. The therapeutic effect through the prevention of "cytokine storm" and T-cell apoptosis. There are several approaches to improve c-ATP. Most of them are easily available through a change in lifestyle. First, regular exercise improves mitochondrial respiratory capacity through an increase in PGC-1 $\alpha$ [18]. Smoking cessation is the second approach to improve mitochondrial capacity and improvement in c-ATP (as mentioned above). Consuming foods with low specific dynamic action (SDA), as the energetic budget for consuming food, can potentially boost the immune system through improving the c-ATP. In 2016, Luoma et al. demonstrated the effect of low-SDA meals in the up-regulation of the innate immune system in corn snakes [19]. On the other hand, several studies have reported the positive effect of xanthine oxidoreductase inhibitors on c-ATP [6].

\section{Consequences of the hypothesis}

This hypothesis provides a new concept to improve the immune system against COVID-19. It demonstrates how an increase in c-ATP can decrease the effect of COVID-19 on immune dysregulation. Considering the strategies to enhance cellular ATP, improvement of the immune system against COVID-19 is possible. It is hoped that this hypothesis will serve as a stimulus for further investigation into this issue.

\section{Declaration of Competing Interest}

The authors declare that they have no known competing financial interests or personal relationships that could have appeared to influence the work reported in this paper. 


\section{Appendix A. Supplementary data}

Supplementary data to this article can be found online at https:// doi.org/10.1016/j.mehy.2020.109762https://doi.org/10.1016/j. mehy.2020.109762.

\section{References}

[1] Lu R, Zhao X, Li J, Niu P, Yang B, Wu H, et al. Genomic characterisation and epidemiology of 2019 novel coronavirus: implications for virus origins and receptor binding. The Lancet 2020;395(10224):565-74.

[2] Kindler E, Thiel V. SARS-CoV and IFN: too little, too late. Cell Host Microbe 2016;19(2):139-41.

[3] de Wit E, van Doremalen N, Falzarano D, Munster VJ. SARS and MERS: recent insights into emerging coronaviruses. Nat Rev Microbiol 2016;14(8):523.

[4] Prompetchara E, Ketloy C, Palaga T. Immune responses in COVID-19 and potential vaccines: Lessons learned from SARS and MERS epidemic. Asian Pac J Allergy Immunol 2020.

[5] Channappanavar R, Fehr AR, Vijay R, Mack M, Zhao J, Meyerholz DK, et al. Dysregulated Type I interferon and inflammatory monocyte-macrophage responses cause lethal pneumonia in SARS-CoV-infected mice. Cell Host Microbe 2016;19(2):181-93.

[6] Johnson TA, Jinnah HA, Kamatani N. Shortage of cellular ATP as a cause of diseases and strategies to enhance ATP. Front Pharmacol 2019;10:98.

[7] Zhang C, He H, Wang L, Zhang N, Huang H, Xiong Q, et al. Virus-triggered ATP release limits viral replication through facilitating IFN- $\beta$ production in a P2X7-dependent manner. J Immunol 2017;199(4):1372-81.

[8] Seif F, Khoshmirsafa M, Aazami H, Mohsenzadegan M, Sedighi G, Bahar M. The role of JAK-STAT signaling pathway and its regulators in the fate of T helper cells. Cell
Commun Signaling 2017;15(1):23.

[9] Cervantes-Barragan L, Züst R, Weber F, Spiegel M, Lang KS, Akira S, et al. Control of coronavirus infection through plasmacytoid dendritic-cell-derived type I interferon. Blood 2007;109(3):1131-7.

[10] Perl A, Gergely Jr P, Nagy G, Koncz A, Banki K. Mitochondrial hyperpolarization: a checkpoint of T-cell life, death and autoimmunity. Trends Immunol 2004;25(7):360-7.

[11] Wu Z, McGoogan JM. Characteristics of and important lessons from the coronavirus disease 2019 (COVID-19) outbreak in China: summary of a report of 72314 cases from the Chinese Center for Disease Control and Prevention. Jama. 2020.

[12] Desler C, Hansen TL, Frederiksen JB, Marcker ML, Singh KK, Juel Rasmussen L. Is there a link between mitochondrial reserve respiratory capacity and aging? J Aging Res 2012;2012.

[13] Qian F, Wang X, Zhang L, Lin A, Zhao H, Fikrig E, et al. Impaired interferon signaling in dendritic cells from older donors infected in vitro with West Nile virus. J Infect Dis 2011;203(10):1415-24.

[14] Malińska D, Więckowski MR, Michalska B, Drabik K, Prill M, Patalas-Krawczyk P, et al. Mitochondria as a possible target for nicotine action. J Bioenerg Biomembr 2019;51(4):259-76.

[15] Jin J-M, Bai P, He W, Wu F, Liu X-F, Han D-M, et al. Gender differences in patients with COVID-19: Focus on severity and mortality. medRxiv. 2020.

[16] Kassi E, Moutsatsou P. Estrogen receptor signaling and its relationship to cytokines in systemic lupus erythematosus. Biomed Res Int 2010;2010.

[17] Zhou F, Yu T, Du R, Fan G, Liu Y, Liu Z, et al. Clinical course and risk factors for mortality of adult inpatients with COVID-19 in Wuhan, China: a retrospective cohort study. The Lancet 2020.

[18] Burelle Y, Hochachka PW. Endurance training induces muscle-specific changes in mitochondrial function in skinned muscle fibers. J Appl Physiol 2002;92(6):2429-38.

[19] Luoma RL, Butler MW, Stahlschmidt ZR. Plasticity of immunity in response to eating. J Exp Biol 2016;219(13):1965-8. 